\title{
Comparison of Different Techniques to Minimize the Dispersion
}

\author{
Ankita Kabra \\ GITS Udaipur (Raj.), India.
}

\author{
Anurag Paliwal \\ Department of Electronics and \\ Communication Engineering, \\ GITS, Udaipur (Raj.), India.
}

\author{
Kiran Rathore \\ GITS, Udaipur (Raj.), India.
}

\begin{abstract}
In this paper, different dispersion compensation techniques are used to compensate the positive dispersion. Three schemes Pre-compensation, Post-compensation, Symmetrical-compensation of DCF, Electronic equalizer of dispersion compensation with DCF and on FBG are proposed. The simulated transmission system has been analyzed on the basic of different parameters by using OptiSystem 7.0 simulator. The results of these dispersion compensation methods are compared in terms of different parameters, which are Q-factor, BER, Eye height and threshold value, investigated at the receiver end. Further, it has been observed that the system needs proper matching between EDFA gain and the length of the fiber for the optimum performance.
\end{abstract}

Keywords: Dispersion compensation, Dispersion compensating fiber (DCF), Electronic Equalizer, Fiber Bragg Grating (FBG), BER, Q-factor, Eye height.

\section{INTRODUCTION}

The structure of an optical fiber includes the core and cladding in which the optical cover is led by the internal reflection method. Light encounters factors such as weakness, destruction and changes while passing the fiber. In this project we deal with the simulation of the random dispersion compensation in fiber. In optical fibers, the wave length creates a temporal widening in dispersion pulses. As the pulse moves in the fiber, it starts to widen and lose its basic forms. One of the suggestions for compensating the dispersion is the dispersion compensation fibers which were widely used in random dispersion. Chipper Bragg gratings are recently used for random dispersion compensation of the fibers. Fiber optic communication is a method of transmitting information from one place to another by sending pulses of light through optical fiber. The light forms an electromagnetic carrier wave that is modulated to carry information. The potential bandwidth of optical communication systems is the driving force behind the worldwide development and deployment of light wave system [1].

Like other communication systems optical communication system also faces problems like dispersion, attenuation and non-linear effects that lead to deterioration in its performance. Among them dispersion affects the system the most and it is tougher to overcome it as compared to other two problems. Thus, it is important to work out an effective dispersion compensation technique that leads to performance enhancement of the optical system.

In this paper different compensation techniques and the comparison between them have been presented.

\section{DISPERSION COMPENSATION TECHNIQUES}

The dispersion compensation is the most important feature to remove the spreading of optical or light pulse in optical fiber communication system.
The most frequently used techniques for dispersion compensation are as follows.

\section{Dispersion Compensating Fibers:}

The use of dispersion compensation fiber is an efficient way to upgrade installed links of standard single mode fiber. Dispersion compensating fibers have a high negative dispersion -70 to $-90 \mathrm{ps} / \mathrm{nm} . \mathrm{km}$ and used to compensate the positive dispersion of transmission fiber.

According to the relative positions of DCF and single mode fiber the three dispersion compensation schemes (pre-DCF, post-DCF and symmetrical/mix-DCF) are proposed. DCF-pre dispersion compensation is achieved by placing the DCF before the standard SMF. Post-DCF scheme achieve dispersion compensation by placing DCF after the standard SMF. Symmetrical/mix-DCF dispersion compensation scheme consists of both pre- and post-DCF dispersion compensation [6].

\section{Electronic Equalizer Technique:}

It is a very attractive technique to compensate for dispersion at the electrical part of the receiver to the transmitter. It is a simple technique that doesn't need any changes in optical transmitting or receiving and also doesn't have considerable loss [4]. Any network changes or adding new devices in the network can be done easily because of adaptive capability of electronic compensator. But there are some disadvantages of this system, for example circuits have limitation in speed compare to optical ones. There are various techniques for using an electronic equalizer, such as: Feed Forward Equalizer (FFE), Feed Forward-Decision Feed Back Equalizer (FFE-DFE), Non Linear Feed Forward- Decision Feedback Equalizer (NL-FFE-DFE) and Maximum Likelihood Sequence Estimator (MLSE) [2]. 


\section{Fiber Bragg Grating:}

Optical Fiber Bragg Grating (FBG) has recently found a practical application in compensation of dispersionbroadening. In this, Chirped Fiber Grating (CFG) is preferred. CFG is a small all-fiber passive device with low insertion loss that is compatible with the transmission system and CFG's dispersion can be easily adjusted. CFG should be located inline for optimum results. This is a preferred technique because of its advantages including small footprint, low insertion loss, dispersion slope compensation and negligible non-linear effects. But the architectures using FBG is complex[3].

\section{SIMULATION SETUP AND DESIGN CONSIDERATION}

We have taken a binary source and encoded it using a NRZ pulse.The optical source used is a CW Laser. Light is used as the carrier and modulated using the Mach Zehnder modulator. The signal is pre amplified using EDFA optical amplifier as the signal has a wavelength of $1550 \mathrm{~nm}$. No inline amplification is used.

The simulations of three dispersion compensation schemes are shown in Fig. 1.a, 1.b, 1.c. In these schemes Data source produces a pseudo random sequence of bits at data rate of 20 Gbits/sec. The output of the data source is given to modulator driver which produces a NRZ format pulse. The output of the laser source is CW type at frequency 193.1 $\mathrm{THz}$ and output power of $5 \mathrm{dBm}$. The Mach-Zehnder modulator has the excitement ratio of $30 \mathrm{db}$. The loop control system has one loop. Each span consists of $250 \mathrm{~km}$ of SMF and $50 \mathrm{~km}$ of DCF in order to fully compensate for the dispersion slope and accumulated dispersion in transmission fiber. The total length of the fiber channel is $300 \mathrm{~km}$. Two EDFAs in front of transmission fiber (SMF) and DCF with gain $25 \mathrm{db}, 20 \mathrm{db}$ respectively and $4 \mathrm{db}$ noise figure. At the receiver side the optical signal transformed into electrical signal by a PIN photodiode, which has $1 \mathrm{~A} / \mathrm{W}$ responsivity and $10 \mathrm{nA}$ dark current. Then the electrical signal is filtered by low pass Bessel filter.

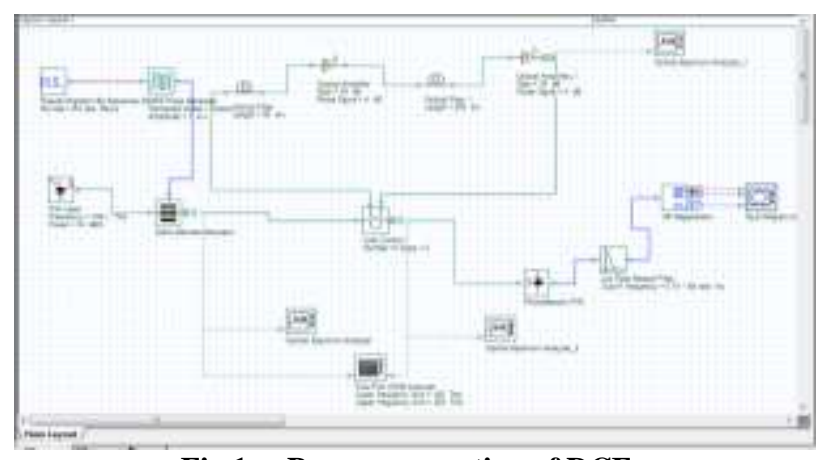

Fig.1.a. Pre compensation of DCF

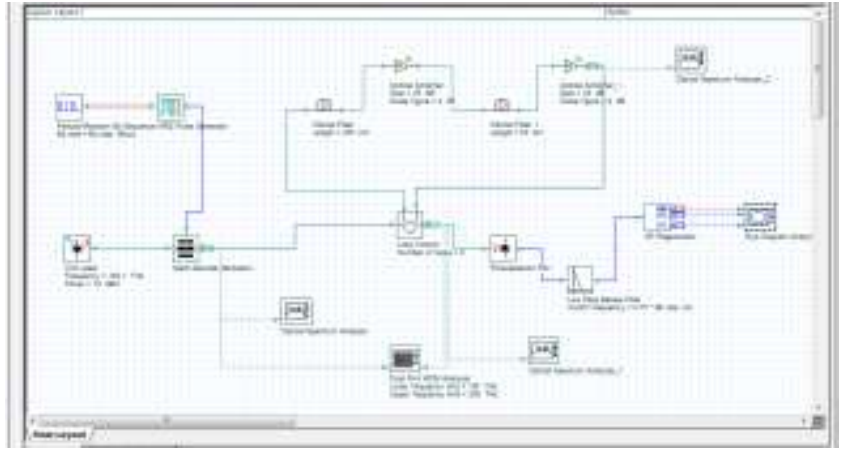

Fig.1.b. Post compensation of DCF

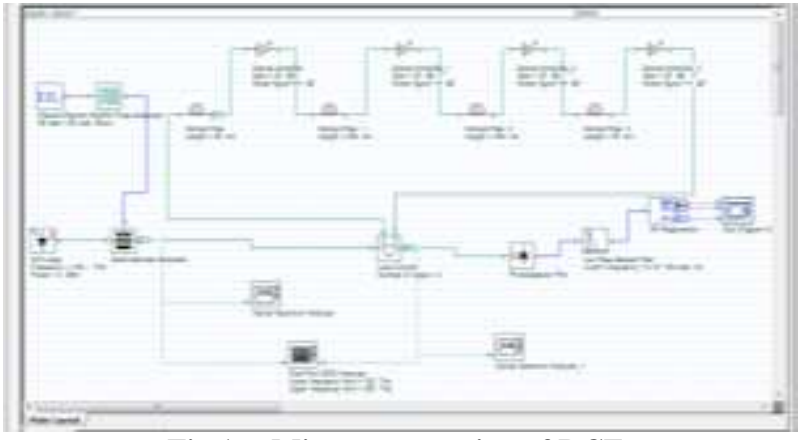

Fig.1.c. Mix compensation of DCF

The next scheme of simulation is to use electronic equalizer [2]. The goal of the simulation is to determine how the number of taps, the resolution of the coefficients, step size and the leakage factor the affect equalizer performance. The motive is to use the outcome of the simulations to establish a feasible hardware implementation. The model used in the simulations is a Optisystem simulation of a fiber optic system with a continuous wave $1550 \mathrm{~nm}$ laser, a standard optical fiber of length $50 \mathrm{~km}$ and a detector in the form of a avalanche diode followed by a low pass filter (to remove high frequency noise) at the receiver[5]. The electronic equalizer scheme is shown in fig. 2.

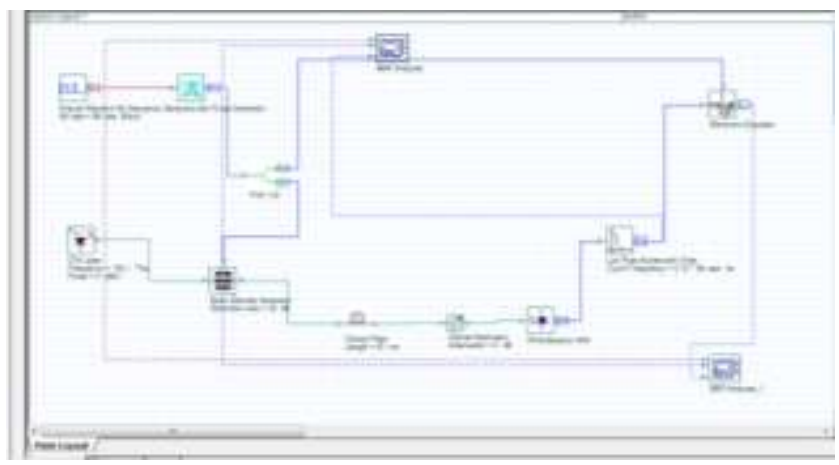

Fig.2 Simulation setup by Electronic Equalizer

The next simulation technique is Fiber Bragg Grating. This is the most important feature of the Bragg gratings which is intensified during the wave length and reflects toward the source and other wave length pass inside without weakening. A chirp in Bragg grating is a kind of chirped that creates changes in the grating period. As the grating period changes along with the axis, different wave lengths are reflected. 
Final effect of a compression is in the input pulse which can be accumulated for dispersion compensation in the telecommunication links [3]. Using the fibers of dispersion compensation needs having them inserted in specified intervals with negative dispersion coefficient in a telecommunication link for removing the dispersion effect of the normal fibers. This simulation scheme is shown in fig.3.

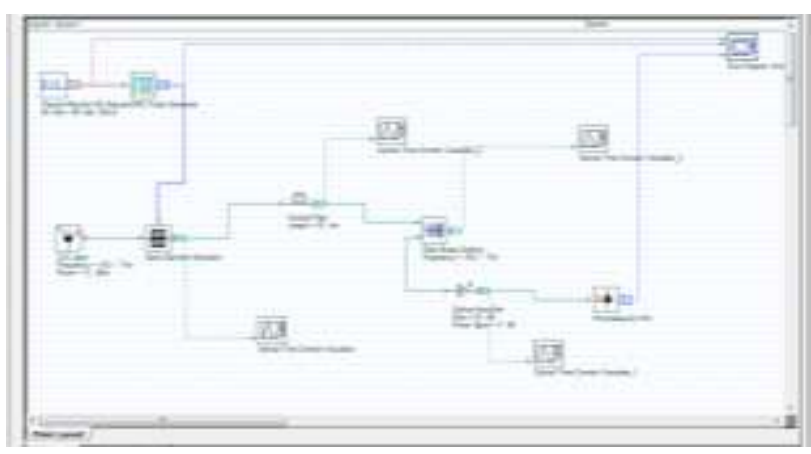

Fig.3 Simulation setup by FBG

\section{RESULTS}

There are larger no of variable and hence large no of possible variable settings in simulations, it is almost impossible to carry out simulation of each and every possible combination within a specific amount of time. The technique utilized here is to change one parameter at a time, analyze the effects and use these analyses to narrow down the scope of the variables to reasonable amount, keeping hardware considerations in mind. The simulations are performed not for a particular equalizer configuration, rather for different possible solutions with different hardware limitations, results are discussed for a variety of possible implementations. To get an idea of the equalizer capabilities, it may be useful to visualize the performance in the form of eye-diagrams.

The simulations are done in Optisystem 7.0 simulator. The eye diagrams for the three DCF schemes are shown in Fig.4.a, 4.b, 4.c.

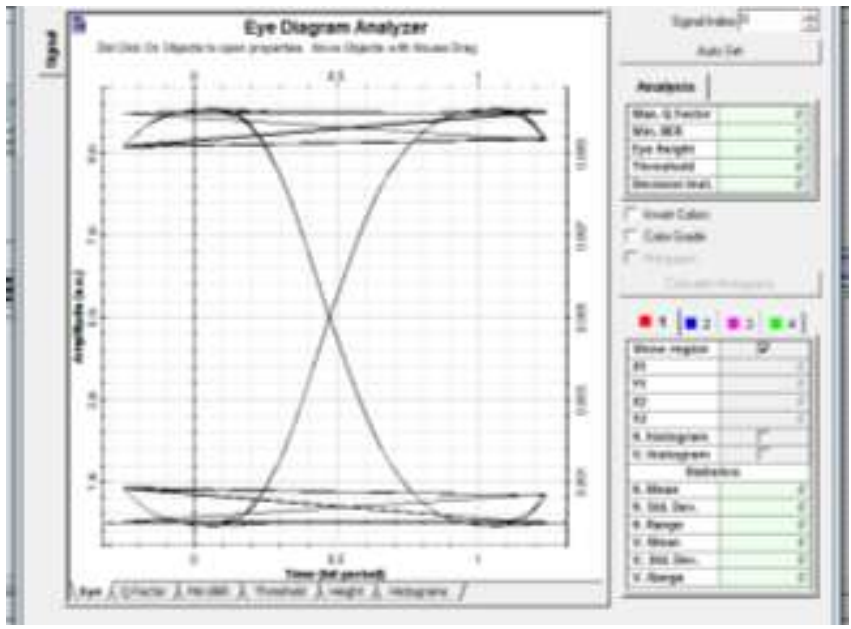

Fig.4.a. Eye diagram of Pre Compensation of DCF

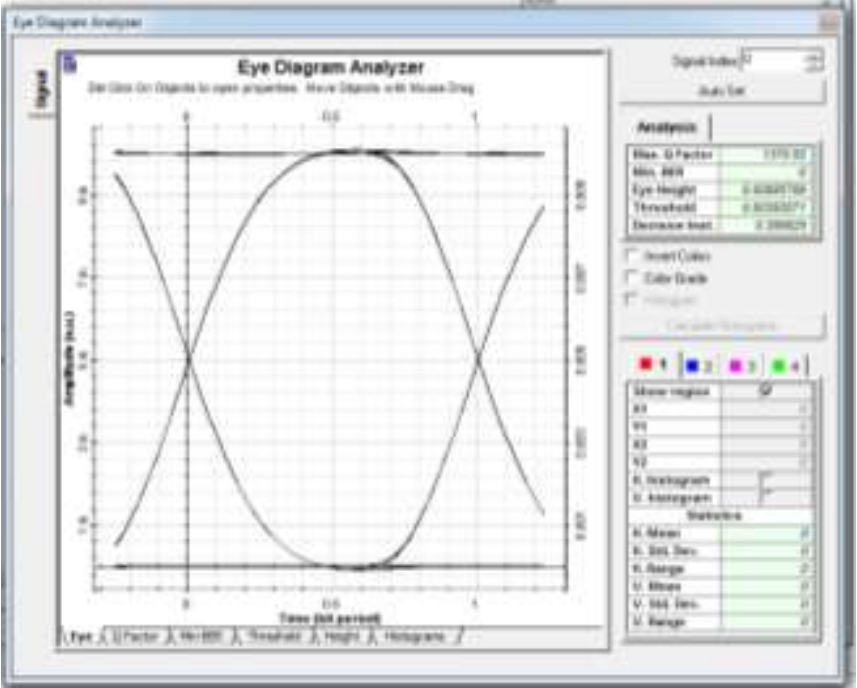

Fig.4.b. Eye diagram of Post Compensation of DCF

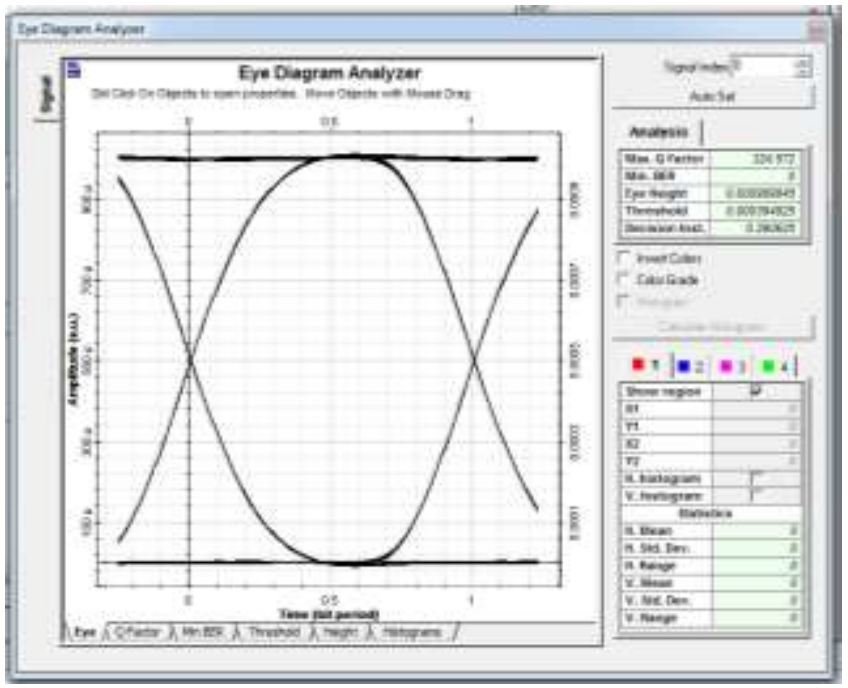

Fig.4.c. Eye diagram of Mix Compensation of DCF

Fig.5 shows the eye diagram of Electronic Equalizer. In this Fig. the BER of the transmitted pulse is obtained with minimum dispersion.

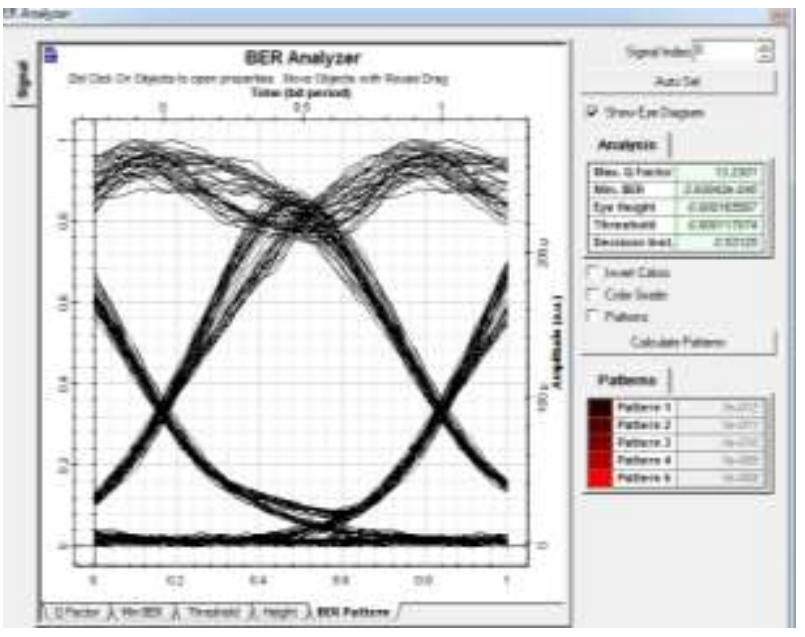

Fig. 5 Eye diagram of Electronic Equalizer 
Fig.6 shows the reflection spectrum and eye diagram of FBG for the designed systems. As it is shown in the figure, the pulse is revived and the power reduction can be compensated.

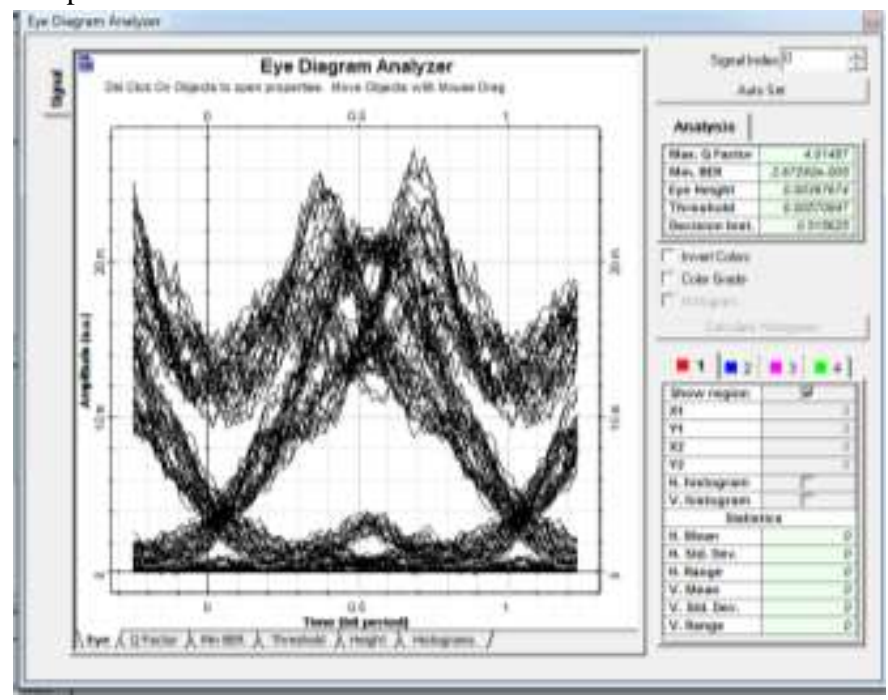

Fig.6 Eye diagram of FBG

The parameters such as, Q-factor, Min. BER, Eye height and Threshold value, for all the three dispersion compensation schemes, electronic equalizer and FBG are tabulated into "Table 1" and compared.

\begin{tabular}{|c|l|l|l|l|}
\hline & $\begin{array}{l}\text { Q- } \\
\text { factor } \\
(\mathbf{d B})\end{array}$ & Min BER & Eye Height & $\begin{array}{l}\text { Threshold } \\
\text { Value }\end{array}$ \\
\hline $\begin{array}{c}\text { Pre } \\
\text { Compensation }\end{array}$ & 1084.64 & $\begin{array}{l}7.79899 \mathrm{e}- \\
028\end{array}$ & 0.00090336 & 0.00026405 \\
\hline $\begin{array}{c}\text { Post } \\
\text { Compensation }\end{array}$ & 1370.52 & $\begin{array}{l}1.64538 \mathrm{e}- \\
023\end{array}$ & 0.00995789 & 0.00393071 \\
\hline $\begin{array}{c}\text { Mix } \\
\text { Compensation }\end{array}$ & 324.572 & $\begin{array}{l}3.04749 \mathrm{e}- \\
044\end{array}$ & 0.000989845 & 0.000394925 \\
\hline $\begin{array}{c}\text { Electronic } \\
\text { Equalizer }\end{array}$ & 13.2301 & $\begin{array}{l}2.93842 \mathrm{e}- \\
040\end{array}$ & 0.000165597 & 0.000117074 \\
\hline FBG & 35.2446 & $\begin{array}{l}1.28591 \mathrm{e}- \\
272\end{array}$ & 0.0861831 & 0.00589062 \\
& & & \\
\hline
\end{tabular}

Table 1: Comparison of dispersion

compensation schemes

From the table and from eye diagrams we can see that the mix compensation scheme and electronic equalizer scheme is much better than all the other compensation schemes.

\section{CONCLUSION}

Dispersion compensation is the optical telecommunication systems is a very challenging and important issue. Without compensating the dispersion, each signal in widened and covers the points near each other so that it cannot be recognized in the receiver.
We have analyzed dispersion compensation with dispersion compensating fibers (DCF) at $20 \mathrm{Gbits} / \mathrm{sec}$ for $250 \mathrm{~km}$ of $\mathrm{SMF}$ and $50 \mathrm{~km}$ of DCF. Different schemes of dispersion compensation like pre-, post-, and symmetrical/mixcompensation with DCF, Electronic equalizer and FBG are proposed in this paper. After analysis, we find that the symmetrical/mix-dispersion compensation scheme and Electronic equalizer scheme is better than pre and postcompensation schemes and FBG scheme. To obtain better signal at receiving end, we check for different combinations of SMF length, DCF length and EDFA gain.

This paper analyses the performance of an Electronic Equalizer to compensate dispersion in a $2.5 \mathrm{Gbps}$ optical fiber link. From the above discussions it is concluded that Electronic Equalizer is indeed an effective way to compensate dispersion in a time dispersive optical link. By carefully manipulating various parameters, the distorted signal can be equalized for even higher data rates fiber links.

As it is shown the length of the dispersion compensation fibers is more and so the nonlinear effects appear and make problems. So using these fibers increases the general loss, nonlinear effects and the costs of the optical transmit systems. In addition, the amount of compensation depends on the wave length and can act in a narrow form. The most important advantages of Electronic Equalizer as compared to other suggested cases of internal loss include the nonlinear effects and low costs.

\section{REFERENCES}

[1] Mehtab Singh, "Different Dispersion Compensation Techniques in Fiber Optic Communication System : A Survey", International Journal of Advanced Research in Electronics and Communication Engineering (IJARECE) Volume 4 Issue 8, August 2015.

[2] Dr. Pulidindi Venugopal, Y.S.V.S.R.Karthik, Jariwala Rudra A, "10Gbps Optical Line Using Electronic Equalizer and its Cost Effectiveness", International Journal of Engineering and Technology (IJET) Vol 5 No 4 Aug-Sep 2013.

[3] Ajeet Singh Verma, A. K. Jaiswal, Mukesh Kumar, "An Improved Methodology for Dispersion Compensation and Synchronization in Optical Fiber Communication Networks", International Journal of Emerging Technology and Advanced Engineering Volume 3, Issue 5, May 2013.

[4] Liuyang Dong, Bo Xu, "Optimization Analysis of Transmission Performance of 10Gb/s optical Signal Using Adaptive Decision Feedback Equalizer", International Conference on Communications and Mobile Computing, 2009. CMC '09. WRI

[5] Edem Ibragimov, "Limits of Optical Dispersion Compensation Using Linear Electrical Equalizer", IEEE PHOTONIC TECHNOLOGY LETTERS, VOL.18, NO.13, JULY 1, 2006.

[6] Manpreet Kaur, Himali Sarangal, "Analysis on Dispersion Compensation with Dispersion Compensation Fiber (DCF)", SSRG International Journal of Electronics and Communication Engineering (SSRG-IJECE) - volume 2 issue $\mathrm{Feb}$

2015 